\title{
Axiological concepts of journalistic texts (lingua- stylistic analysis)
}

\author{
Assel Kozhakhmetova - Zhanna Ospanova - Manat Mussatayeva - Zhanat \\ Bissenbayeva
}

DOI: 10.18355/XL.2020.13.02.19

\begin{abstract}
The description of the axiological concepts of the journalistic texts are related to solving the issues of media-linguistics, and axiological linguistics, while also providing insight to solve the pragmatic problems of linguistics itself. Values are embodied in language and consciousness in the form of axiological concepts and, together with archetypal concepts, form part of the axiological conceptosphere of an individual or community. In turn, axiological and archetypal concepts are updated against the background of axiological strategies that form a discursive space and determine the deployment of certain communicative scenarios. Integration processes in modern science determine the appeal of linguists to axiological concepts and methods in the study of the text. The urgency of the topic is due to several factors. Media texts are of interest to researchers since they are distinguished by stylistic multidimensionality and variability. Scientists are actively arguing about the correlation of such functional and cognitive phenomena as a media course, a media text, a journalistic style, a style of mass communication, etc. Linguists talk about the merging of social discourse with the media discourse about the mediation of journalism. Therefore, the relevance of this work lies in the development, expansion and deepening of scientific research of media texts as a communicative, cognitive and functional-stylistic phenomenon, in the creation of an invariant model of the axiological structure of the text, which provides the opportunity for effective study and description of the object, and in the development of metalanguage of linguistics. The aim of the research is to describe the axiological component of a newspaper text.

Key words: axiological, concepts, journalistic, texts, lingua- stylistic, analysis
\end{abstract}

\section{Introduction}

A journalistic text is a structurally and systemically organized, communicative, iconic unit, which is a holistic and complete message, functioning in the field of mass communication, reflecting social issues. The media text is at the center of scientific research of various nature and content, as it is material evidence, a product of mass communication, stores information about the extra-linguistic context, about verbal and other codes of communication. The claims of linguistics on this specific object of study are explained by the fact that the text is the focus of all the mechanisms of language and speech, all the secrets of the embodiment of human thought in the clothes of the word.

Media communication can be considered as a collection of texts that perform various functions. Moreover, texts cease to be just a tool, as they "are being created more and more not to express and transmit actual ideas and truly new truths, but with the goal of increasing the quantity of oneself" (Lukin, 2006).

The text reflects, stores, and conveys the values of the human individual and the human person himself/herself is a value. The text captures and translates estimates that themselves become the object of evaluation in the context of naive or scientific knowledge. Studying the assessments and values recorded in journalistic texts allows one to reach the level of two models: the text model (as a relatively independent, self-valuable, and self-reproducing system) and the model of value consciousness of the person who creates and receives these texts.

XLinguae, Volume 13 Issue 2, April 2020, ISSN 1337-8384, ISSN 2453-711X 
Values are an integral part of the picture of the human world, an extremely significant social phenomenon, as well as the basis for both unity and disunity of people and societies. It is thus natural that the problem of values is of increasing interest among linguists today. The axiological side of oral and written communication is becoming one of the most important factors in the study of various phenomena in linguistics. Values have, in recent decades, become the subject of modern lingua-axiological studies and are considered within the anthropocentric paradigm that has been established in linguistics as a linguistic category.

Thus, it seems that linguistic interest in values arises because of their wide representation in the language on the one hand, and, as noted above, due to the correspondence of values to the most important parameters that allow them to be classified as a linguistic category, on another. The existing diversity of the main directions of the study of values in domestic and foreign linguistic studies reflects the complex nature of values and indicates interest in each of the parameters that this phenomenon possesses. So, we consider it logical to consider, first of all, the cognitive parameter of values. Attributing value meaning to something, or, on the contrary, the decision that it is "something" devoid of it, is the result of human gnostic and cognitive activity, which, in turn, determines speech patterns of behavior. Cognitive processes in the individual's mind provide scientists with additional opportunities and a unique perspective, which more fully reveals the specifics of the embodiment of values in language and discourse. Values in the framework of cognitive axiology are studied by integrating axiological views from other disciplines, such as philosophy, psychology, and pedagogy, into linguistics. The goal of this approach is to analyze how leading or frequent values are encoded into a specific discourse and used in various areas of the language for special purposes [Lago, 1996]. Understanding how the linguistic consciousness of speakers of different cultures and languages is arranged also allows us to identify the general and individual traits in their perception and processing of reality.

According to this research paradigm, most of the concepts lie in the world of intangible entities, which are the result of the conceptualization of the person. Concepts such as love, friendship, tradition, motherhood, and many others, including value concepts proper, do not exist outside of a person's experience, and their understanding is dependent on a person's disposition. All these concepts are the results of cognitive processes and, in particular, conceptualization processes (Krzeszowski, 1997).

V. I. Karasik, therefore, considers values from various points of view, including as an integral part of the discourse, calling values, along with other parameters, the fundamental characteristics of institutional discourse (Karasik, 2002). We cannot disagree with the observations of T. A. Shiryaeva who argues that when modeling the institutional frames of business discourse, the scientist defines professional dominants as the key socio-cultural professional component of the discourse, which is characterized by mental constancy, semantic integrity, high derivational potential and non-uniformity (Shiryaeva, 2013).

Scientists who develop the issue of updating values at the lexical level agree that values can be objectified through a wide variety of language units. So, the English scientist R.M. Hare considered values in the vein of prescriptivism, according to which words that verbalize values have both descriptive and prescriptive meanings. The function of the first value is to convey information about the object to which the word is applied, the function of the second is to recommend and highlight a specific object among the class of similar objects. According to R.M. Hearu, estimated language structure alone has the goal of forming a certain attitude, judgment, impact (Hare, 1967).

Another approach to the study of values in scientific research of a social orientation boils down to the consideration of values as a factor in the flow of 
institutional communication. P. Bourdieu and G. Becker, analyzing the social formation of aesthetic ideas and values, conclude that any socially significant object or phenomenon is the cooperation of more than one figure in the framework of certain social institutions (Bourdieu, 1987; Becker, 1984).

Thus, values are a complex linguistic phenomenon, the most complete study of which requires a combination of approaches and scientific directions. Such an analysis of values provides great opportunities for describing and studying the linguistic consciousness of a nation, for building a model of a linguistic personality, and for reconstructing a model of one or another discourse, an integral component of which is the value preferences embodied in the language.

One of the most promising approaches to the study of values in linguistic research is the close symbiosis of cognitive-discursive and axiolinguistic approaches. It seems that values exist in language and consciousness in the form of axiologically loaded concepts, the study of which today is logical to carry out through conceptual and discourse analysis. These approaches allow us to identify the signs and content of axiological concepts, especially their language update. Just as all concepts of a language are combined into a conceptual sphere, axiological concepts are also organized into a system - an axiological conceptual sphere, or axiosphere, which has its own structural features.

The modeling of the axiosphere involves the identification of its components at the level of language and thinking. It seems that the axiosphere can be individual or public, depending on whether it is about the values of an individual or the whole community, including a professional one. As the study showed, along with axiological concepts, archetypal concepts that objectify sustainable knowledge about the world, which are embodied through repeating plots, motives, and scenarios, should be included in the axiosphere.

Archetypes have great axiological potential, since they are not criticized by the native speaker of the language and culture, and excite a number of stable images in the consciousness of the participant in the discourse. Finally, the axiological background of the discourse is created through the implementation of a number of axiological strategies - a combination of language tools and techniques aimed at updating axiological and archetypal concepts, as well as the formation and direction of communication. So, the phenomenon of values is the result of pragma-cognitive activity of a person and determines the way of his thinking, determines certain patterns of behavior.

The scientific description of the assessment category reflects and structures the results of a naive conceptualization of the world. Moreover, any scientific model tends to invariance, accepting and assuming as inevitable the fact that there is some conceptual variability. For science, the search for optimal ways of speech impact on the audience remains relevant, on the one hand; on the other hand, the creation of tools to identify the author's intentions is also considered important.

So, the relevance of this work lies in the development, expansion and deepening of scientific research of media texts as a communicative, cognitive and functional-stylistic phenomenon, in the creation of an invariant model of the axiological structure of the text, which provides the opportunity for effective study and description of the object, in the development of metalanguage of linguistics.

The aim of the research is to describe the axiological component of a newspaper text. This goal involves solving a number of research questions:

What are the linguistic tools for describing the axiological nature of the

text?

What are the structure valuation vectors in a media text? 
(identify the specifics of the content, form, nature, orientation, degree of explicitness of the assessment, methods of implicating evaluative statements, language means of euphemizing)

During the research of axiological concepts of journalistic texts, we firstly decided to explain and analyze what is a text by itself. We did a lot of analyses and came up with different approaches to the denotation of the text. The text remains the focus of modern science. In the scientific and educational literature, various definitions of the text are systematized, and reviews of approaches to its study are given (Kazarin, 2003; Boguslavskaya, 2008; Zalevskaya, 2008). Despite all the differences, the common key point in the definition of a text is an indication of its orderliness, organization, systematic nature, and it is structural. As an example, we give several definitions of "consistent sign construction (implemented according to the rules of some sign system), forming a holistic, complete message" (Krasnoyarova, 2002), "a set of rules of linguistic and extralinguistic organization of the content of communication of representatives of a certain linguistic and cultural community" (Prokhorov, 2009), "a whole complex functioning as a structural-semantic unity" (Turaeva, 2009). Journalistic text understood by us as (1) ordered, organized, (2) a communicative, symbolic unit, (3) representing a holistic and complete message, (4) functioning in the field of mass communication, and (5) reflecting a certain topic.

To describe the organization of the text in linguistics, the terms system and structure are traditionally used. Structure and consistency are immanent properties of any text. A journalistic text is a combination of internal structures or systems figurative, stylistic, thematic, narrative, communicative, axiological, etc.

The concept of a text structure is sometimes equated with the concept of a text system in the meaning of a certain construct, structure, or organization of an object. When delimiting the terms, the system is associated with the idea of the object of study as a combination of any elements, including relations (paradigmatic aspect), the structure is analyzed as a mechanism, action, implementation of these elements, first of all, their connections (Bolotnova, 2005). The idea of the structure of the text as a "global way of organizing an object as a holistic reality" (Turaeva, 2009) is based on the concepts of structural linguistics and structuralism in literary criticism. All scientists who have studied the text are M. P. Brandes, V. V., I. R. Halperin have approached this idea to one degree or another.

In domestic and foreign science, various types, types of text structures are described: linguistic (syntactic, lexical, etc.), compositional, speech, semantic, semantic, communicative, conceptual, cognitive, etc. Text structures are classified into simple, complex, highly complex, internal and external, linear and nonlinear, deep and surface.

At the same time, two points of view on nature coexist in the science of text structures. The first is connected with the idea of the inner life of the text. Its adherents tend to analyze the structure without being in touch with the author and recipient of the text, "absolutize" the text, describe the structure of the text as a synergistic process, declare structural self-organization speech works, see (Moskalchuk, 2003). Others dispute such point of view and do not recognize the text as a "self-sufficient entity," consider that "the body of the text, taken by itself, without a person who means it, does not carry any internal energy, cannot be organized structurally" (Zalevskaya, 2008).

So, the structure of the text is the organization of relations of its elements (linguistic and extralinguistic nature) vertically and horizontally in accordance with the author's intention and interpretation procedures of the addressee. Understanding the text as a result of speech activity emphasizes the static aspect of the structure of the text. At the same time, the main feature of the structure is its mobility and ability to change in quantitative and qualitative plans. Mobility, due to the position of the 
observer (researcher) of the text, features his consciousness, an individual picture of the world in which the text is stored as a concept.

In order to make a research on the issue of axiological concepts of journalistic texts, we selected different text from media and analyzed them from the linguistic and stylistically point of view. Firstly, in the following part of the research paper, we demonstrate the methods of the research.

\section{Methods}

As a method of axiological research, a description of stereotypes of discourse, various types of implications, categories of norms, and assessments, which serve as methods of objectification of values, has been used (Babaeva, 2003).

At the stage of generalizing the material, the mathematical method is used as the main one, and the material is processed using the n-factorial formula. At all stages of scientific research, the descriptive method and introspection technique, which consists in referring to the linguistic intuition of the researcher in the process of linguistic modeling, have remained relevant.

During the study from 2016 to 2019, a lot of newspaper materials were analyzed, but in this study, we present examples specifically for 2018 and 2019 from January to December. The analyzed material will be presented later in the practical part of this article in the form of graphs. For the indicated period, 168 texts of 9600 sentences were studied and considered.

\section{Discussion}

Axiological components of the text are composed of values represented by sign systems, including linguistic and textual mechanisms. The most relevant values for media texts are the subject of the study of axiology (Shilov, 2005). Today, axiology has moved from the stage of self-determination to the stage of integration with other sciences. The axiosphere acts as an object of axiology. This term is used "to denote the world of values in any sense" (Stolovich, 1999)

Table No. 1 designations of understanding of values.

\begin{tabular}{|l|l|l|}
\hline № & name & kinds \\
\hline 1 & world of values & may be subjective, but may exist \\
\hline 2 & value consciousness & $\begin{array}{l}\text { representations, grades, tastes, ideals, } \\
\text { norms, }\end{array}$ \\
\hline 3 & creative results & canons, samples \\
\hline
\end{tabular}

As part of our analysis of the corpus of fragments of a newspaper text, we highlighted several dominant semantic expressions of values and construction of expressions based on professional value representations of addressees and serving to activate axiological meanings in the text: for example, the axiological component of the text is expressed by phrases of consent $(38.2 \%$ of the total number of texts analyzed), phrases of patriotism (24.2\%), by means of phrases of tolerance (17.4\%), and some were analyzed shows a text saying the phrase by means of education $(20.2 \%)$ (see Figure .: 1). 


\section{Analysis of axiological concepts expression in publicistic texts}

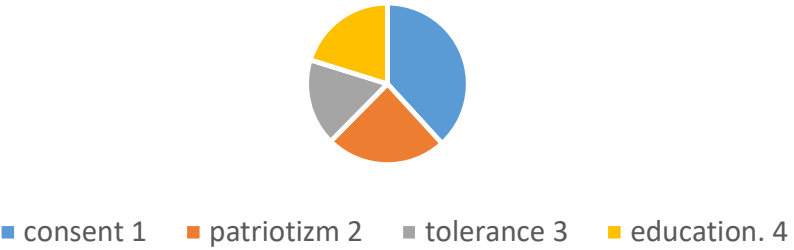

Along with the verbalization of axiological and archetypal concepts, the modern text of the newspaper implements a number of axiological strategies through which the identified concepts are embodied.

The study of the axiological characteristics of a newspaper text, in our opinion, is impossible without the study of these strategies since they put all the cognitive-linguistic tools used in the media text into a single integrated system.

We believe that the study of speech strategies from the point of view of axiological linguistics allows us to more accurately answer the questions "why?" and "how?" professional addressees who form the newspaper's text create texts that are attractive to readers.

As a result of the analysis of theoretical scientific research concerning the specifics of speech strategies, as well as considering the results of practical processing of empirical material, it was found that a single component that combines all axiologically labeled strategies is the communicative goal of influence, which, in turn, is designed to form a positive attitude towards the most important value dominants in the recipient and to facilitate their integration into the axiological system.

Ways to achieve this goal are to activate and form the most important human landmarks, embodied in the form of values and archetypes, as well as to use a complex of speech actions, which include assessment, high speech emotion, stylistic techniques, and stylistically colored vocabulary.

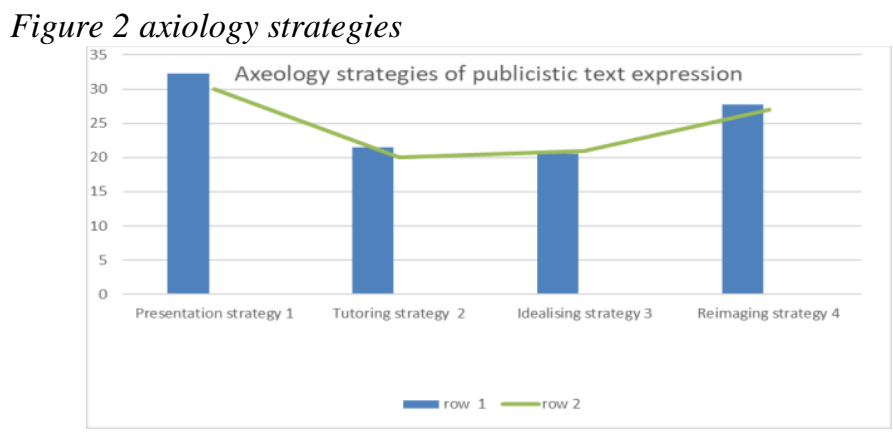

Each of these strategies highlights a particular thematic and pragmatically determined situation and unfolds as a specific set of linguistic and extralinguistic means that affect the perception of the addressee.

The axiological component of strategies, at the same time, is embodied not only in the fact of using concepts embodying the values of the professional 
community, and archetypal concepts used intentionally in the texts, but also manifests itself in the form of axiologically defined attitudes. The latter, in our opinion, involve not only the motivation of the addressee to take any action but also the integration of a particular model of behavior into the individual's value system. The most common axiological strategy identified in the newspaper text is the strategy presentation, found in $32.3 \%$ of articles analyzed during the study.

Thus, the fragments from the newspaper that we analyzed inform the reader about the widely-publicized event in the camp form a certain image and also encourage a certain assessment. The combination of linguistic and cognitive tools used by the author as part of the presentation strategy contributes to the perception of this text as a holistic news message containing an implicit appeal.

\section{Conclusion}

Axiological picture of the world as part of a holistic picture of the world fragmentary displayed in texts, forming relatively independent (closed) value system axiological field text. This is an axiological field. Obeying the laws of creation, functioning, decoding, and interpretation of a text is embodied in its axiological structure that is the result of interpretative activities of the author and reader. Any media and political text, regardless of genre affiliation and idiostyle, has an axiological structure. We can say that the condition for the appearance of the axiostructure is the very fact of the existence of the text and the subject creating it.

The specificity of the axiological structure is determined by a number of extralinguistic and linguistic factors. The newspaper text, becoming a fact of mass communication, is "saturated" with the functional, stylistic, pragmatic characteristics of the media.

The significance of the media in the life of modern man is determined by the creation of a media picture of the world. For the interpretation and analysis of newspaper texts, it becomes important to have a holistic view of the media picture of the world, which "appears as a kind of mosaic of private discursive options that differ thematically, media, etc." (Rezanova, 2008). Researchers come to the following conclusions about the media picture of the world: 1) the linguistic picture created in the discourses of the media gives an almost complete picture of the picture of the world that dominates in the mass consciousness; 2) among other factors (the individual's life experience, interpersonal communication and the activities of social institutions - churches, schools, etc.) that influence the formation of the picture of the world, the media are the dominant factor; 3) media information forms an information picture of the world, which in the media interacts with the linguistic a picture of the world; 4) as a result of the impact of the media in the recipient's picture of the world special cognitive structures are created, the mental-cognitive result of the interaction of communicants becomes important; 5) the assessment of the world through modeling the picture of the world is the media's most important task; 6) the purpose of the media impact is the general (nuclear) part of the individual's worldview; 7) the media are changing the individual picture of the world, but at the same time, individual pictures do not become unified; the media picture of the world has a regulatory function; 8) mass communication creates a special reality and a special subculture; 9) modern culture is to a large extent a product of the mass media system. The findings are not certain. So, some scholars argue that mass media do not create, but destroy culture (Luman, 2005), that "the effects of mass communication are most clearly manifested in the field of informing, rather than changing attitudes" (Boguslavskaya, 2008). Given the right to the existence of various views and concepts, one has to recognize the intuitively felt powerful influence of the media on the minds of modern cultural bearers. The pragmatics of media discourse determine 
the "subordination" of the axiological structure to the goals of ideological influence on the mass recipient.

The study of the axiological field of newspaper texts, including the application of the axiological model proposed in the framework of this work, allows us to make several important generalizations and conclusions, which, in our opinion, must be considered when axiological analysis of objects in the linguistic-stylistic aspect.

Vertical and horizontal organization of values, their composition, the quantity, degree, and methods of codification form the specifics newspaper discourse. In the center of the political axiosphere there are legal, moral, and ethical values. Nuclear media texts reflect the conceptual system offered to the mass addressee as their value orientations, patterns, norms. The concept, value, and word form an axiological triad, which reveals the differences between philosophical entities, linguistic-cognitive and verbal units. An axiological concept is a cognitive construct that concentrates knowledge, ideas, concepts about the basic ethical, aesthetic, social, and other kinds of values - will and freedom, righteousness and sin, truth and truth, etc. Evaluation and evaluation are distinguished as potential and relevant. Evaluation is the potential of a language unit, its ability to explicate the positive or negative properties of an object, its fixation on the evaluation axis, its place in the axiological field.

Assessment is the mental-verbal action of assigning positive or negative properties to one or another object. The value enshrined in the consciousness of a native speaker as an axiological concept or value (evaluative) component of another conceptual unit acts as the basis for evaluation and is itself the object of evaluation. Value and its actualization are never reduced to the act of evaluation.

Values and assessments are inextricably linked with cognitive and verbalmental phenomena - prototypes, stereotypes, norms. Prototypes are the basis for frames, scripts, concepts, gestalt. Prototype images are realized in axiologically marked, figurative nominations; prototype schemes are expressed at the semanticsyntactic, compositional levels, in the structure of the narrative.

The stereotype-image or stereotype-situation is the result of the interpretation of the corresponding prototype through the prism of social norms. Stereotypes are delimited from such cognitive constructs as a concept, frame, script, script, by a number of criteria: content, form, nature, degree of intuition.

Stereotypes are included in the axiological picture of the world, can be interpreted as values, and become the basis, motivation for evaluation. Social assessments are stereotyped in nature due to the dominance of the collective. The norm-standard as a positive assessment of the images and schemes of any objects and / or phenomena is fixed (or ready for implementation) in verbal form. The normstandard approaches the stereotype, as it preserves the naive idea of the "normal" (ordinary) state of affairs. Positive cultural values are interpreted as norms. A norm can acquire the status of value if it is declared as a goal or is fixed on the reclamation axis ( $\mathrm{X}$ is the norm and $\mathrm{X}$ is good). Normative statements are a special case of value judgment, serve the purpose of value argumentation, explicate the position of a collective author, broadcast ideological and other assessments.

The practical significance of the work lies in the fact that its results can be used in the practice of training linguists, journalists, strategists, and other specialists in the field of communication. Practical material and theoretical conclusions of the thesis can be reflected in the courses of text linguistics, stylistics, lingua-culturology, cognitive linguistics, and the language of the media.

A description of the mechanism for realizing the axiological potential of a media text, a number of compositional and stylistic techniques for enhancing the credibility of evaluation, euphemization, and dysfemization, creating a repertoire of 
value-marked scenarios and axiological roles play the role of landmarks in journalistic and linguistic practice in achieving the optimal pragmatic effect in linguistics.

\section{Bibliographic references}

BABAEVA, E. V. 2002. Normative and evaluative concepts of German and Russian linguistic cultures. In: Reality, language and consciousness. Vol. 2. Tambov: Publishing house of TSU, pp. 379-386.

BABAEVA, E. V. 2003. Discursive measurement of values: a monograph. Volzhsky. $102 \mathrm{p}$.

BOGDANOVA, I. A. 2006. The functioning of the archetypal concept of "water" in the texts of folk and individual creativity: abstract. dis. ... cand. filol. Sciences: 10.02.19 / Bogdanova Irina Aleksandrovna. Perm. 24 p.

BARNARD, M. 2002. Fashion as Communication. Second Edition In: M. Barnard. London; New York Routledge. 210 p.

BECKER, H. S. 1984. Art Worlds. In: H.S. Becker. Berkeley University of California Press. 392 p.

BOGUSLAVSKAYA, V. V. 2008. Text modeling: linguasociocultural concept. Analysis of journalistic texts. Publishing House of LCI. 280 p.

BOURDIEU, P. 1987. Distinction: A Social Critique of the Judgment of Taste In: P. Bourdieu. Cambridge: Harvard University Press. 640 p.

BOLOTNOVA, N. S. 2009. Communicative style of the text: vocabulary thesaurus. Flint: Nauka. 384 p.

BRANDES, M. P. 2006. Criticism of translation: Workshop on stylistic-comparative analysis of translations of German and Russian literary texts. KDU. 240 p.

BRANDES, M. P. 2009. Stylistic analysis: Based on German material. Librocom. 208 p.

HALPERIN, I. R. 2006. Text as an object of linguistic research. KomKniga. 144 p.

HEAR, P. M. 1985. Description and evaluation. In: New in foreign linguistics. vol. 16. Linguistic pragmatics. Progress, pp. 183-195.

KARASIK, V. I. 2002. Language of social status. Gnosis. 333 p.

KARASIK, V. I. 2004. Language personality: perspectives of linguistic personology. In: Language personality as a subject of theoretical and applied linguistics. Tula: Publishing house of TulSU. pp. 92-99.

KARASIK, V. I. 2004. Language circle: personality, concepts, discourse. Gnosis. 392p.

KRASNOYAROVA, O. V. 2002. The structure of the journalistic text. Irkutsk: Publishing House of BSUEP. 208 p.

KRZESZOWSKI, T. P. 1997. Angels and Devils in Hell. Elements of Axiology in Semantic - T.P. Krzeszovwski. Warsaw: Energeia. 298 p.

LAGO, F. A. 1996. Axioematic analysis of brand names in English: A semanticopragmatic approach to branding. In: F.A. Lago - Multilingualism in Specialist Communication (Proceedings of the 10th European Symposium on Language for Special Purposes) / Gerhard Budin (ed.). - Vienna: IITF In: Infoterm, vol. II., pp. 991-1010.

LUKIN, V. A. 2006. Crisis and text. Russian Word in the Russian World - 2005: State and Statehood in the Linguistic Consciousness of Russians. pp. 126-167.

LUMAN, N. 2005. Reality mass media / trans. with him. A.Yu. Antonovsky. Praxis. $256 \mathrm{p}$.

MARKELOVA, T. V. 1993. Assessment and appraisal. In: The semantic structure of words and utterances. pp. 107-115.

MOSKALCHUK, G. G. 2003. Text structure as a synergistic process. URSS editorial. 296p.

XLinguae, Volume 13 Issue 2, April 2020, ISSN 1337-8384, ISSN 2453-711X 
PAVLIKOVA, M. 2018. The power of modern technologies in the fiction of Don DeLillo. In: Communications - Scientific Letters of the University of Zilina, vol. 20, 1PartA, pp. 57-60. ISSN 1335-4205.

PROKHOROV, Y. E. 2006. Reality. Text. Discourse. Flint: Nauka. 224 p.

REZANOVA, Z. I. 2008. Modern institutional discourses: interpretive activities of the addressee. In: Commentary and interpretation of the text. Novosibirsk: Publishing House of NGPU, pp. 201-214.

STOLOVICH, L. N. 1994. Beauty. Good. Truth: Essay on the history of aesthetic axiology. Republic. 464 p.

SHIRYAEVA, T. A. 2013. Institutionality as the most important discourse-forming factor. In: T. A. Shiryaeva - Man. Tongue. Culture: Sat scientific Art. dedicated to the 60th anniversary of prof. IN AND. Karasika. Kiev: Publ. house of D. Burago, Part 1, pp. 780-790.

SHIRYAEVA, T. A. 2009. Language as a means of constructing social reality. In: T.A. Shiryaeva - Language. Text. Discourse. Stavropol: Publishing House of the State Pedagogical Institute. n. 7. pp. 61-67.

TURAEVA, Z. A. 2009. Linguistic text. Text: Structure and semantics. URSS, pp. 144.

ZALEVSKAYA, A. A. 2006. Mental lexicon from the standpoint of different approaches. In: Actual problems of modern linguistics. Flint: Nauka. pp. 311-326.

Words: 5043

Characters: 33183 (18,44 standard pages)

Assel Kozhakhmetova

Abay Kazakh National Pedagogical University

Department of Russian Language and Literature

Dostyk ave 13, Almaty

Kazakhstan

Zhanna Ospanova

L.N.Gumilyov Eurasian National University,

Foreign Philology Department

st. Satbayeva 2, Almaty district, Nur-Sultan

Kazakhstan

professor Manat Mussatayeva

Abay Kazakh National Pedagogical University

Department of Russian Language and Literature

dostyk ave 13, Almaty

Kazakhstan

associate professor Zhanat Bissenbayeva

al Farabi Kazakh National university

Temiryazeva street 71, Almaty

Kazakhstan

zhanat_2006@mail.ru 